Revista Educación 25(1): 27-33, 2001

\title{
REFLEXIONES TEÓRICO-METODOLÓGICAS PARA DESARROLLAR EL PROCESO DE INDUCCIÓN COMO APOYO A LA GESTIÓN DEL RECURSO HUMANO UNIVERSITARIO
}

Víctor Hugo Orozco Delgado

\begin{abstract}
Resumen: Se analizan en este artículo elementos básicos que deben ser considerados en el proceso de gestión del recurso bumano universitario. Se ofrece especial atención al funcionario universitario que por primera vez inicia sus labores, sobre todo, por la ansiedad, nerviosismo y otras situaciones que enfrenta cuando ingresa por primera vez a una organización tan grande y compleja en sus relaciones como lo es la universidad..

Se presenta a los responsables de dirigir y administrar el recurso humano universitario, algunas estrategias que les permitan generar confianza y seguridad entre quienes laboran a su alrededor y con ello, contribuir sin duda al logro de las metas organizacionales.
\end{abstract}

\section{Introducción}

La gestión de recursos humanos debe ser una de las tareas prioritarias en la formación de la estrategia de la organización universitaria. Comprende la integración de las personas a la gestión estratégica, el compromiso de éstas con los planes formulados y su orientación hacia la calidad del desempeño académico o administrativo. Constituye la gestión de recursos humanos un área de interés fundamental en las universidades costarricenses. Dentro de esta gestión, algunos factores que deben ser de importante consideración, son los siguientes: el entorno, que incluye no solo la creciente competencia que se ha desatado entre las instituciones de educación superior, así como también las oportunidades laborales que se ofrecen dentro del ámbito universitario; el aumento en tamaño y complejidad de las universidades, lo que implica mayor demanda de mano de obra, la limitación en sus recursos y principalmente la necesidad de comprometer y trabajar con la gente, como alternativa que la lleve al éxito.

En relación con este último elemento, puede afirmarse que cualquier proceso de gestión de recursos humanos tiene particular importancia dentro de una determinada organización, por cuanto se relaciona directamente con las personas con las cuales se trabaja; a las que es necesario conocer en cuanto a sus necesidades, deseos, expectativas; de 
manera tal que contribuyan al logro de los objetivos y metas de la organización. Esto no siempre se logra, entre otras cosas por no planificar sistemáticamente, o porque no se tiene convencimiento de los planes y proyectos establecidos, porque las personas no se involucran con éstos, porque surgen contradicciones entre los diferentes niveles de la organización o finalmente porque los actores involucrados en el proceso de gestión de recursos humanos no se sienten satisfechos con el trabajo que realizan.

Preocupado por el recurso humano y sobre todo porque en instituciones como la Universidad de Costa Rica o el Instituto Tecnológico de Costa Rica, no se tienen claramente definidas políticas que orienten y reciban al personal que por primera vez se incorpora a dichos centros de trabajo, he considerado conveniente reflexionar en torno a un tema que a mi juicio es relevante y que en alguna medida podría contribuir a elevar el rendimiento y el grado de satisfacción que hacia el trabajo tienen los funcionarios universitarios. Me refiero específicamente al proceso de inducción como alternativa para incorporar, de manera positiva, a los funcionarios a su trabajo.

Consultas hechas en la Oficina de Recursos Humanos de la Universidad de Costa Rica y a funcionarios del CEDA (Centro de Desarrollo Académico) del Instituto Tecnológico de Costa Rica, dan cuenta de que en la mayoría de las diferentes unidades académicas que conforman dichas organizaciones, no se tienen políticas claras en cuanto a la inducción de los nuevos funcionarios.

En primera instancia, es importante destacar el hecho de que todo lo bueno que la universidad haga, se construye bajo el aporte y fortaleza de su recurso humano; éste, por lo tanto, debe ser bien atendido desde el momento mismo en que inicia su trabajo. Pero para mejorar el rendimiento de este valioso recurso humano es necesario tener claro que su proceso de administración debe ser a largo plazo e implica consecuentemente un compromiso con el cambio, el cual puede lograrse dando participación en el proceso mismo, ofreciendo esfuerzos positivos para lograrlo, minimizando sorpresas, compartiendo información y sobre todo ofreciendo a la alta gerencia su propio compromiso de cambio. Las unidades académicas y quienes están al frente, podrán lograrlo en la medida en que entiendan que el cambio las obliga a innovar, crear y sobre todo crecer con su recurso humano; ver el cambio como una actividad permanente. Una de las acciones encaminadas a realizar dicho cambio, es la atención que debe darse al funcionario que se contrata por primera vez, para que desde el primer momento en que inicie sus funciones perciba y viva un ambiente agradable de trabajo, que le permita a su vez ofrecer un servicio de calidad en el mismo.

Es necesario entender que la primera impresión de un funcionario acerca de la empresa o institución donde laborará, tiene relación directa con las circunstancias bajo las cuales se instalará en ella, el trabajo que desempeñará y las relaciones funcionales con el resto del personal.

Se considera que una de las principales razones por las que las personas cambian de trabajo, se debe fundamentalmente a que no se sienten bienvenidas ni se perciben como parte de la organización a la que se adhieren; o se dan cuenta de que la institución o el trabajo no son lo que esperaban (Cadwell, 1991).

La universidad, por su parte y como organización formal, requiere la imperiosa necesidad de que todo funcionario que inicie sus labores, ya sea de índole administrativo o docente, conozca de la mejor manera la organización y éste a la misma, de manera tal que le permita a ambos, familiarizarse e integrarse entre sí. Dicho cometido será posible si se implementa, ejecuta y retroalimenta un adecuado proceso de inducción de personal.

El éxito del funcionario implica también el éxito de la organización. Una adecuada inducción y sobre todo su óptima planificación puede ser el primer paso en el camino hacia el logro de éste. 


\section{El proceso de inducción como apoyo a la gestión del recurso humano universitario}

Se refiere la inducción a un proceso encaminado a introducir o presentar a los nuevos empleados de una determinada organización, ofreciéndoles asistencia y apoyo; de manera que les permitan familiarizarse lo antes posible con ella y que conozcan entre otras cosas, el clima de la organización, los servicios que ofrece, la distribución de los espacios físicos y el detalle de las funciones y puestos.

Se pretende con dicho proceso, reducir el nivel de ansiedad y nerviosismo a que se exponen los funcionarios al ingresar por primera vez a su lugar de trabajo, ofrecerles la nueva información que necesitan para funcionar en forma cómoda y eficaz dentro de la organización, además, ayudarlos en la comprensión de los aspectos específicos de su trabajo, facilitarles los aspectos mínimos de procedimientos, organización, marco jurídico laboral propio de la organización y finalmente, promover la adaptación de sus características personales con los demás trabajadores y la cultura organizacional.

La debida orientación del nuevo servidor le permitirá adaptarse a las exigencias de la organización y contribuir eficaz y eficientemente al logro de los objetivos individuales y organizativos.

Se constituye en un proceso que reviste gran importancia por cuanto la orientación del servidor (docente, misceláneo, guarda, oficinista, secretaria, jefe de unidad, otros) se convierte en el camino por el cual el funcionario se integrará de la mejor manera a sus funciones, a su grupo de trabajo y a la organización en general. Probablemente las necesidades básicas de seguridad, pertenencia, estima y reconocimiento, se satisfacen en esta fase de incorporación del servidor a la organización. Por ello, decanos, directores de unidades, entre otros, deben poner especial atención a esta etapa.
Es importante indicar que todo individuo es único y aunque en alguna medida esté debidamente acreditado para desempeñar un puesto, no debe dejarse de lado la necesidad de proporcionarle la información básica para que se adapte de la mejor manera a la organización. Debe procurarse la disminución de la ansiedad que se genera en los primeros días de trabajo y procurar ante todo el fortalecimiento de las relaciones de la organización con su fuerza de trabajo. Por su parte, (Dessler, 1991) afirma que el fortalecimiento de esta relación podrá incrementar el compromiso de la persona con la organización y asegurar una conducta consistente y predecible al moldear las actitudes y valores de los empleados de acuerdo con los de la institución.

Puede afirmarse, entonces, a manera de síntesis, que un proceso de inducción de personal conlleva una serie de ventajas para la institución, entre las cuales, según Mendoza (1985:36), se podrían enumerar: la facilidad para establecer la integración entre el personal y la institución, la reducción del tiempo que normalmente tarde el trabajador en conocer las características de la organización y sus labores, la oportunidad de ofrecer a los trabajadores una visión general sobre los productos o servicios de la organización y les permite conocer la forma en que su labor encaja dentro de ésta y la facilidad para aplicar los métodos de capacitación que se puedan emplear después de la inducción; estas ventajas naturalmente están encaminadas a vencer los problemas habituales que generalmente enfrenta todo funcionario cuando se incorpora por primera vez a la organización, a las cuales se hizo referencia anteriormente.

Además de los aspectos enumerados, debe tenerse presente que las expectativas que un funcionario docente o administrativo tenga de la organización y la satisfacción o no de éstas, determinará, en alguna medida, el grado de satisfacción que se logre hacia el trabajo realizado. 


\section{Papel del administrador universitario ante el proceso de inducción del personal}

Para que el proceso de inducción tenga el éxito deseado, se requiere en la unidad o dependencia ejecutora, como se afirmó anteriormente, un administrador comprometido con el cambio, con visión de futuro, que propicie una activa y responsable participación del recurso humano propio de la organización. Un administrador que promueva el crecimiento continuo del recurso humano, que facilite el establecimiento de un clima organizacional saludable, en el cual se mantengan positivas relaciones interpersonales. Un clima en el cual se fomente el trabajo en equipo, la cooperación, la participación en la toma de decisiones, la responsabilidad, la fluidez en la comunicación, entre otros; como elementos que coadyuven al logro de las metas organizativas.

Se requiere de un administrador líder, con capacidad para interpretar y atender las exigencias internas y externas de la organización. Con capacidad y habilidad para trabajar eficientemente con los demás y percibir cambios en el comportamiento de los trabajadores, en sus necesidades humanas. Un líder que coordine, combine y guíe los esfuerzos de quienes conforman la organización, que innove y sobre todo que la oriente hacia los cambios esperados. Sobre todo, un líder que logre converger las fortalezas individuales en aras de objetivos comunes (López, 1999).

\section{Determinación de estrategias por utilizar durante el proceso de inducción}

Para llevar adelante un adecuado proceso de inducción, el director de una facultad, departamento, unidad académica u otros, puede recurrir a diversas estrategias, para lo cual debe considerar el tamaño de la organización y la disponibilidad de recursos y espacios para tal fin.
A continuación se presentan algunas estrategias que podrían contribuir al desarrollo óptimo del programa de inducción.

a. Entrevista del decano, director, jefe de unidad, otros, con el nuevo servidor. Este le explicará entre otras cosas, detalles específicos del puesto, problemas y posibles soluciones que pudiese enfrentar en un momento determinado.

b. Visita explicada a las instalaciones que conforman el edificio universitario, cuando sea posible. Esto le permitirá al trabajador ubicarse físicamente dentro de la planta y oficina, conocer diversos lugares e instancias a las que tendrá que acudir en caso necesario.

c. Entrega de un manual de bienvenida, en el cual se incluya información básica y recomendaciones más importantes al servidor de nuevo ingreso.

d. Conferencia o reunión general con el personal al que se dirige el proceso, la cual puede incluir la presentación de un invitado especial, audiovisual, etc.

e. Curso, seminario o taller de integración de la organización, el cual puede realizarse periódicamente durante los ciclos lectivos.

Es recomendable que el programa que se elabore para tal efecto considere entre otros los siguientes aspectos:

En relación con la organización: su historia, políticas, trayectoria, ubicación, recursos disponibles, servicios que ofrece, distribución de espacios físicos, otros.

En cuanto a los funcionarios y la administración: Horarios de trabajo, funciones, control de asistencia, otros.

$Y$ en cuanto al proceso de enseñanza $y$ aprendizaje debe entenderse que la docencia es una de las áreas fundamentales de la universidad y que por lo tanto sus esfuerzos deben dirigirse a ésta, sin dejar de lado aspectos esenciales de investigación y acción social. Información oportuna sobre este particular, debe consultarse en documentos como: 
Reglamento del Régimen Académico y Servicio Docente, Políticas y Normas Curriculares, Políticas y Procedimientos de Evaluación del Desempeño, Régimen Disciplinario, Estatuto Orgánico y normativas que establecen las vicerrectorías de docencia, entre otros.

La definición de las estrategias presentadas anteriormente, le permitirán al responsable de desarrollar el proceso de inducción, solventar algunos de los problemas comunes que se presentan cuando se implementa un programa de inducción; como pueden ser: la posibilidad de que no se tenga claridad de los beneficios que un programa de esta naturaleza pueda generarle a la organización, así como la poca colaboración y apoyo de los demás miembros del personal hacia quienes se incorporan por primera vez a sus funciones, la falta de colaboración de los coordinadores de departamento, unidad, otros, para lograr una integración eficiente de los nuevos funcionarios a su puesto; además, el implementar un programa de inducción deficiente, alejado de la realidad de la institución, lo que podría causar resentimiento y frustración en el nuevo funcionario y finalmente, ejecutar el programa de manera rápida e improvisada, sin considerarlo como un proceso gradual debidamente planificado. Surge entonces la necesidad de que el programa de inducción se elabore con la premura debida, previendo para ello el tiempo y los recursos necesarios, así como la posibilidad de realizar los ajustes pertinentes para su correcta ejecución. En relación con el tiempo, Maya, (1996) considera que el proceso debe iniciarse tan pronto como el nuevo funcionario se incorpore a la organización, una vez claramente definidas las condiciones de su nombramiento. Al respecto es muy importante que el decano, director de unidad, otros, se tomen el tiempo necesario para que el nuevo servidor se sienta seguro y bienvenido. El primer día de ingreso es trascendental y debe insistirse en la necesidad de estar preparado para este momento, en el sentido de evacuar y resolver, con la seguridad que el puesto lo exige, cualquier duda o inquietud que los funcionarios presenten y requieran solventar, ya sea en relación con la institución o comunidad en que se encuentra inmersa ésta. Las cosas deben empezar correctamente y sobre todo tener la clara convicción de que el proceso no es sólo un día: es continuo y progresivo.

\subsection{Evaluación del proceso de inducción}

Es importante que el administrador de la unidad o sobre quien recaiga la responsabilidad de desarrollar el programa de inducción, realice un análisis minucioso, no solo de la evaluación de los nuevos empleados, sino también de su propia autoevaluación (ver instrumentos en la sección de anexos) para que se fortalezca y mejore lo que haya salido bien o llenado las expectativas y si no fuese así, tomar las medidas pertinentes y hacer los ajustes necesarios para mejorar el proceso, por el beneficio mismo de la organización.

\section{Referencias bibliográficas}

Cadwell, Charles. Inducción del nuevo empleado. México: Trillas, 1991.

Dessler, Gary. Administración de Personal. México: Prentice-Hall Hispanoamericana S.A., 1991.

López, Olimpia. "La gestión del centro educativo de enseñanza primaria y el desarrollo bumano en el contexto de fines de siglo". En: Revista de Educación, vol. 23, No. 2, 1999.

Mendoza, Alejandro. La capacitación práctica en las organizaciones. México: Trillas, 1985.

Maya, Arnobio. "Separata" Simed en Acción. San José: Año VI, No 1, 1996.

Stoner y Otros. Administración. México: Prentice-Hall Hispanoamericana S. A., 1996. 
ANEXOS

\section{Evaluación}

Recuerde cuando se inició en su trabajo actual. Lea cada afirmación y encierre en un círculo C (cierto) o F (falso) según se relacionen con la inducción que usted recibió. (Adaptado de M. Cadwel 1991:19).

\begin{tabular}{|c|c|c|}
\hline 1- Me hicieron sentir bienvenido. & $\mathrm{C}$ & $\mathrm{F}$ \\
\hline 2- Me presentaron a los demás miembros de mi grupo de trabajo. & $\mathrm{C}$ & $\mathrm{F}$ \\
\hline 3- Mi director me puso atención y me dio la bienvenida. & $\mathrm{C}$ & $\mathrm{F}$ \\
\hline 4- Mi inducción pareció estar bien planificada. & $\mathrm{C}$ & $\mathrm{F}$ \\
\hline 5- Hice una visita a la organización con una persona calificada. & $\mathrm{C}$ & $\mathrm{F}$ \\
\hline 6- Aprendí sobre la historia de la institución y planes futuros. & $\mathrm{C}$ & $\mathrm{F}$ \\
\hline 7- Me quedaron claramente definidas las funciones y las tareas de mi puesto. & $\mathrm{C}$ & $\mathrm{F}$ \\
\hline 8- Conocí los servicios básicos que ofrecía la institución. & $\mathrm{C}$ & $\mathrm{F}$ \\
\hline 9- Conocí a mis compañeros de departamento o unidad. & $\mathrm{C}$ & $\mathrm{F}$ \\
\hline 10- Tuve la oportunidad de hacer preguntas. & $\mathrm{C}$ & $\mathrm{F}$ \\
\hline 11- Se me asignó el aula o lugar de mi trabajo. & $\mathrm{C}$ & $\mathrm{F}$ \\
\hline 12- Recibí información básica sobre equipos, materiales, etc. relativos a mi trabajo. & $\mathrm{C}$ & $\mathrm{F}$ \\
\hline $\begin{array}{l}\text { 13- Se me definieron claramente las condiciones económicas de mi contrato o } \\
\text { nombramiento. }\end{array}$ & $\mathrm{C}$ & $\mathrm{F}$ \\
\hline 14- Pude compartir con mis compañeros de trabajo. & $\mathrm{C}$ & $\mathrm{F}$ \\
\hline 15- Se me hizo una asignación específica de trabajo junto con sus instrucciones. & $\mathrm{C}$ & $\mathrm{F}$ \\
\hline 16- Conocí el horario institucional. & $\mathrm{C}$ & $\mathrm{F}$ \\
\hline 17- Conocí mis deberes, prohibiciones y derechos laborales. & $\mathrm{C}$ & $\mathrm{F}$ \\
\hline 18- Conocí y participé de los diferentes comités de trabajo de la institución. & $\mathrm{C}$ & $\mathrm{F}$ \\
\hline 19- Conocí los procedimientos para permisos, incapacidades, licencias. & $\mathrm{C}$ & $\mathrm{F}$ \\
\hline 20- Al final de la primera semana me sentí como miembro de la organización. & $\mathrm{C}$ & $\mathrm{F}$ \\
\hline
\end{tabular}

Total de C

Total de $\mathbf{F}$

¿Cómo califica su inducción?

18-20 Ciertas: Su inducción fue sobresaliente

15-17 Ciertas: Su inducción estuvo arriba del promedio. Puede hacer algunas mejoras.

11-14 Ciertas: Desafortunadamente usted recibió una inducción típica. Debe hacerse mucho por mejorarla.

10 o menos: Debe replantearse el proceso de inducción. No permita que le pase lo mismo a otros. 
Para que el director o responsable autoevalúe el proceso de inducción realizada en su institución a cargo, se propone lo siguiente (adaptación de Arnobio Maya, Op. cit).

¿En qué proporción fueron cubiertas las expectativas de una buena inducción?

\begin{tabular}{|r|l|}
\hline 1. & Presentación con los demás. \\
\hline 2. & Recorrido por las instalaciones. \\
\hline 3. & Simpatía de los compañeros y las compañeras. \\
\hline 4. & Disposición del supervisor y usted mismo. \\
\hline 5. & Organización del área de trabajo. \\
\hline 6. & Primera asignación de trabajo. \\
\hline 7. & Explicación sobre el funcionamiento de la Escuela, Unidad. \\
\hline 8. & Ayuda en la institución del nuevo docente o empleado. \\
\hline 9. & Explicación de la importancia de trabajar en su organización. \\
\hline 10. & Inducción adecuadamente planificada. \\
\hline
\end{tabular}

Aplique esta escala a cada uno de los puntos expuestos anteriormente.

5. Excedió sus expectativas.

4. Cubrió sus expectativas.

3. Cubrió parcialmente sus expectativas.

2. Dejó mucho que desear.

1. Falló sus expectativas. 